Cinémas

Revue d'études cinématographiques

Journal of Film Studies

Prédal, René. Le Cinéma français depuis 1945. Paris : Nathan, 1991, 568 p.

\title{
Éric Fortin
}

Volume 4, numéro 1, automne 1993

URI : https://id.erudit.org/iderudit/1000117ar

DOI : https://doi.org/10.7202/1000117ar

Aller au sommaire du numéro

Éditeur(s)

Cinémas

ISSN

1181-6945 (imprimé)

1705-6500 (numérique)

Découvrir la revue

Citer ce compte rendu

Fortin, É. (1993). Compte rendu de [Prédal, René. Le Cinéma français depuis

1945. Paris : Nathan, 1991, 568 p.] Cinémas, 4(1), 151-155.

https://doi.org/10.7202/1000117ar d'utilisation que vous pouvez consulter en ligne.

https://apropos.erudit.org/fr/usagers/politique-dutilisation/ 
PRÉDAL, René. Le Cinéma français depuis 1945. Paris : Nathan, 1991, 568 p.

Cet ouvrage de référence, divisé en quatre parties principales, chacune d'elles étant composée de quatre ou cinq chapitres, constitue une base solide pour toute étude concernant le cinéma français de l'après-guerre. La bibliographie générale commentée par l'auteur oriente le lecteur vers une quantité considérable de pistes de lecture, concernant l'entière période couverte par le livre, alors que chacune des grandes parties, ainsi que chacun des chapitres, possède sa propre bibliographie «spécialisée» et commentée.

La première partie ( $L a$ qualité française, un cinéma institutionnalisé) couvre, en gros, la période 1945-57, soit celle amorcée avec la libération de la France en 1944, et qui s'étend jusqu'aux prémisses de la Nouvelle Vague. Les combats pour la libération de Paris ayant arrêté production et exploitation, le cinéma français est mal en point et on assiste alors à sa lente et progressive structuration : commissions et comités sont créés, et les organismes syndicaux, revus. Mais les changements sont minces et le cinéma français, malgré quelques cas isolés, et malgré ce passage à la réalisation de quelques nouveaux noms (Bresson, Clouzot, Becker, Cayatte, Clément) durant l'Occupation, incline alors davantage vers un sentiment de continuité qu'il ne tente de créer élans de rupture. Parce qu'ils préfèrent rétablir le climat de stabilité financière qui prévalait avant la guerre, puis restaurer leur syndicat plutôt que de jongler avec les problèmes de langage et d'esthétique, les cinéastesvedettes (Autant-Lara, Carné, Clair, etc.) se fieront encore, pour une bonne décennie, aux formules et recettes éprouvées durant les années 30. Il est vrai que les succès au «box-office» et la reconnaissance qu'obtiennent alors les films français dans les festivals internationaux entretiennent et motivent cet immobilisme des cinéastes de la «qualité française».

Personne ne bouge, hormis les Bresson, Cocteau, et Becker, ou des cas isolés tel Rouquier, ainsi que la poignée sans cesse grandissante de jeunes critiques et cinéastes qui, à coups d'articles frondeurs et lapidaires ou de courts métrages étonnants, commence à transformer le calme paysage du cinéma français.

La seconde partie, intitulée La Nouvelle Vague et le triomphe de l'esthétique des auteurs, traite quant à elle de la période 1958- 
67, soit de l'émergence de la Nouvelle Vague et de son déclin, jusqu'au contexte précédant les tentatives soixante-huitardes. Alors que moins de dix films se distinguaient de ceux de la production dite de qualité durant la décennie 1947-57, la seule saison 1958-59 en compte une dizaine (et la tendance se maintiendra pendant trois ans). C'est l'explosion. La Nouvelle Vague déferle, et ses principales têtes d'affiche ont pour noms Astruc, Vadim, Malle, Chabrol, Kast, Resnais, Truffaut, Rohmer, Godard, Rivette, etc. Les uns sont produits par le système selon les classes habituelles (petit polar, scandale, atout culturel), alors que les autres, bénéficiant de la loi de l'autoproduction et de diverses subventions, travaillent de manière artisanale. On s'autoproduit totalement ou en partie et l'on aide ses copains à faire leurs débuts. L'offensive, d'abord individuelle, de quelques-uns permet l'ouverture d'une brèche dans le système de la production, du syndicat et des institutions étatiques qui bouleversent des règles longtemps immuables. Leur bataille, les cinéastes de la Nouvelle Vague la mènent principalement (et corrélativement) sur deux fronts : elle est tout autant esthétique qu'économique. De modestes budgets, joints à une volonté d'affirmer leur statut d'auteur (avec les Hawks, Hitchcock, Renoir, Cocteau, Bresson, Becker et Rossellini pour exemples), conduisent ces jeunes cinéastes à réaliser des œuvres personnelles, intimes, qui rejettent la technique au nom du réalisme.

Peu de cinéastes des générations précédentes échappent au balayage de la Nouvelle Vague; seul Bresson semble savoir profiter du bouleversement et des ouvertures créées par les plus jeunes pour améliorer les conditions de sa carrière. Les anciens sont chassés et vont poursuivre leur filmographie à la télévision où ils retrouvent l'infrastructure d'antan. Profitant de cet intérêt du public pour la création et l'expression, bon nombre de jeunes cinéastes qui sont cinéphiles et possèdent leur propre démarche personnelle, sans toutefois provenir de la critique et n'entretenir aucune relation avec la Nouvelle Vague, émergent et contribuent à l'ampleur du mouvement.

En quatre ans, le public est inondé de films réalisés par de nouveaux auteurs, et bien que certains d'entre eux font énormément d'entrées, la production est dans l'ensemble plutôt inégale. Au «box-office», les mauvais scores commencent à s'accumuler à une vitesse vertigineuse. Ce flot régulier d'insuccès ne tarde pas à miner la confiance des producteurs. De fait, bien des cinéastes devront attendre plusieurs années avant de faire un second film. Certains se résigneront, d'autres 
aborderont momentanément un virage vers le documentaire ou la pratique du $16 \mathrm{~mm}$ ou se dirigeront vers cette télévision qui, d'années en années, ne cesse de ravir du public au cinéma. En 1965, la Nouvelle Vague est bien éteinte, sans n'avoir véritablement «permis» à une génération intermédiaire de s'imposer. Ses principales personnalités continuent toutefois à s'imposer durant les décennies suivantes, un peu moins pour certains, il est vrai, durant une portion des années 70 , mais cette période difficile n'est en rien comparable à celle que connurent les cinéastes de la «qualité française».

La troisième partie, Remise en question: société, idéologie et cinéma, couvre cette période fort mouvementée du cinéma français comprise entre 1968 et 1980, des événements préfigurant mai en passant par le cinéma militant, le cinéma politique, les légères mutations du cinéma commercial, le virage de certains réalisateurs de la Nouvelle Vague, la venue de quelques nouveaux réalisateurs, les désillusions, jusqu'au retour à un cinéma narratif qui, sans n'avoir jamais été délaissé, n'est désormais plus concevable de la même façon pour certains réalisateurs. Comme ce fut le cas pour l'Occupation, les événements bouleversants de mai 68 sont filmés par quelques cinéastes, mais très peu de films sont achevés. Et bien que Les états généraux du cinéma français provoquent de brillantes discussions et soulèvent d'importants remaniements, la plupart des cinéastes «arrivés» retournent à leurs préoccupations d'avant la révolution; il semble que seuls Godard, Marker et Malle modifient par la suite leur pratique du cinéma. Néanmoins, une grande majorité des jeunes qui n'ont pas encore abordé le long métrage, interrogent et conçoivent différemment le cinéma depuis ces états généraux. Il y a une volonté consciente de faire les choses autrement. Non sans une certaine naïveté, les réalisateurs de la Nouvelle Vague, assurés de faire mieux que leurs aînés, étaient impatients de passer aux actes. Ceux d'après 1968 sont davantage conscients et lucides, et leurs films viennent justement s'inscrire contre le spontanéisme des années 60. Ainsi les Chris Marker, Duras, Eustache, Blain, Straub et Huillet balisent-ils à peu près tout l'éventail des possibles cinématographiques. La plupart des options et des champs expérimentés ne seront pas poursuivis sous leurs formes originelles, mais ils contribuent néanmoins à enrichir un cinéma qui paraît beaucoup plus complexe à la fin de cette décennie qu'à la fin de la précédente.

Les œuvres fortes (Doillon, Pialat, Eustache) ne manquent pas, mais le public boude les salles de cinéma - il est vrai que la 
télévision a remplacé les ciné-clubs comme instrument de culture, et que la critique est plus ou moins emballée. C'est donc ici que le cinéma achève de quitter les devants de la scène des loisirs pour joindre le secteur culturel, domaine protégé où il recevra moins de coups et pourra mieux «digérer ses révolutions successives». Durant la dernière moitié des années 70 , le cinéma narratif hérité de la «qualité française» côtoie l'expression personnelle de la Nouvelle Vague et les préoccupations sociales et politiques d'après 68 .

La quatrième partie, Le septième art, marchandise culturelle ou le cinéma des années 80, traite de la période 1981-91. Le mouvement de chute des fréquentations en salles amorcé au cours des années 70, mais quelque peu ralenti autour de 1977, prend des allures de catastrophe à partir de 1982, la pression de la distribution américaine, l'arrivée en force de la vidéo et la fulgurante ascension de la télévision française en étant les principales responsables. Face à pareille crise, le ministre Lang propose plusieurs réformes visant à donner la priorité aux salles, ainsi qu'il augmente considérablement le budget des divers organismes de subventions. Lang donne beaucoup, mais ne sait à qui donner - aussi est-il généreux avec tout le monde, aidant aussi bien les débutants que les auteurs établis ou les superproductions. Mais la part de coproduction de la télévision ne cesse de croître au fil des ans, imposant avec une plus grande autorité ses normes bas de gamme. En somme, le cinéma français s'est en quelque sorte graduellement "vendu à la télévision, en remplaçant les recettes salles par les achats de diffusion T.V.». Pour pallier à cette crise et concurrencer les Américains, on se lance également dans le gigantisme et les superproductions (L'Ours, Camille Claudel, Jean de Florette ). À l'opposé, le cinéma d'auteur, qui a retenu les leçons de la Nouvelle Vague, verse dans le budget modeste, l'autoproduction, et s'attache aux petits producteurs; il est à ce sujet éloquent de noter que les auteurs tournant le plus au cours de cette décennie sont Godard, Rohmer, Mocky et Doillon. Il est vrai que la vulnérabilité du système et la multiplication des sources de production favorisent ces valeurs sûres. Mais, si bon nombre de jeunes cinéastes (et parmi eux, pour la première fois, une quantité considérable de femmes), soutenus par une Avance sur recettes aux ressources augmentées, parviennent à réaliser un premier long métrage durant cette décennie, il faudra attendre longtemps et souvent en vain l'arrivée de leur second.

Le cinéma français des années 80 est caractérisé par son éclectisme; coïncé entre l'appel d'une certaine nostalgie et la 
comptabilisation de l'acquis du cinéma moderne, il hésite, inclinant selon le penchant de chacun. C'est un cinéma du recyclage, qui poursuit et encourage le métissage des genres amorcé vers la fin de la décennie précédente, tout en étant désormais constitué par une somme de «manifestations individuelles passionnantes», de créateurs isolés qui plus que jamais font triompher la politique des auteurs. Enfin, malgré la force des produits américains et le financement télévisé, l'auteur de ce colossal ouvrage demeure confiant quant à un éventuel redressement du paysage cinématographique français.

$\mathrm{Au}$ sein du très fragile milieu de l'édition des livres sur le cinéma, la maison Nathan et sa collection Nathan/Université se distingue de ses pairs par la constante pertinence de ses auteurs qui, malgré la diversité de leurs intérêts et champs d'étude, produisent des œuvres toujours exemplaires et éclairantes auxquelles une majorité d'universitaires ne peut éviter de réserver une place de choix parmi les ouvrages de référence de leurs bibliographies de travail. Le dernier livre de ce spécialiste du cinéma français qu'est René Prédal, Le Cinéma français depuis 1945, paru chez le même éditeur, ne manque pas de s'inscrire dans la foulée de cette vertueuse tendance, perpétuant avec une égale rigueur, ainsi qu'un égal souci de simplicité et de clarté, la tradition amorcée sur d'autres sujets par les Jost, Gaudreault, Vanoye, Aumont. L'ouvrage est riche, fécond et sonde une quantité imposante d'aspects de cette tranche d'histoire du cinéma français, l'auteur n'hésitant pas à s'appuyer sur l'éventail des sciences sociales, tout en se gardant bien de promouvoir un ton «socialisant».

Éric Fortin

Université de Montréal 